\title{
A novel adsorbent for Sr2+ prepared by grafting the DB18C6 on D564
} resins

\author{
Xiushen Ye $\mathrm{e}^{1, \mathrm{a}}$, Yaoqiang Hu $\mathrm{Hu}^{1,2, \mathrm{~b}}$, Chaoming Quan ${ }^{1,2, \mathrm{c}}$, Li Sun ${ }^{1,2, \mathrm{~d}}$, Haining $\mathrm{Liu}^{1, \mathrm{e}^{*}}$ \\ Zhijian $\mathrm{Wu}^{1, \mathrm{f}}$
}

${ }^{1}$ Qinghai Institute of Salt Lakes, Chinese Academy of Sciences, Xining 810008, China

${ }^{2}$ The Graduate University of Chinese Academy of Sciences, Beijing 100049, China

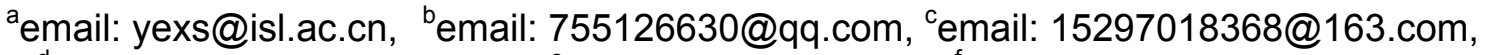

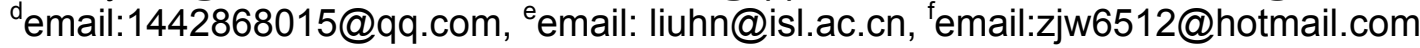

Corresponding Author: Haining Liu

Keywords: 4,4'-Dibromodibenzo-18-crown-6, Dibenzo-18-Crown-6, $\mathrm{Sr}^{2+}$, graft, adsorption

\begin{abstract}
A novel adsorbent was prepared by grafted the Dibenzo-18-Crown-6-Ether (DB18C6) on D564 resins and characterized by Thermogravimetry (TG) and Fourier Transform Infrared Spectroscopy (FTIR). Adsorption of $\mathrm{Sr}^{2+}$ from aqueous solutions was investigated in batch method as a function of contact time, concentration of $\mathrm{Sr}^{2+}$ and $\mathrm{pH}$ of the solution. It is found that the adsorbent has good selectivity for $\mathrm{Sr}^{2+}$. The efficient adsorption of $\mathrm{Sr}^{2+}$ is obtained at $\mathrm{pH}$ 5-6 before adsorption or $\mathrm{pH} 7-8$ after adsorption. And the adsorption amount increases with the $\mathrm{Sr}^{2+}$ concentration and decreases with the temperature of the solution.
\end{abstract}

\section{Introduction}

Strontium and its compounds have numerous industrial applications and they are typical fission products resulting from nuclear reactions[1]. Radioactive ${ }^{90} \mathrm{Sr}$, is one of the most frequently found radionuclides in the soil and groundwater at nuclear weapon test sites and nuclear waste repositories[2]. In addition, because of the chemical similarity to calcium, $\mathrm{Sr}^{2+}$ can easily replace $\mathrm{Ca}^{2+}$ in human bodies and cause anemia, leukemia, as well as other chronic illnesses. Hence, it is very important to concentrate and separate the $\mathrm{Sr}(\mathrm{II})$ ions from the waste solutions[3].

The examined methods for isolation of $\mathrm{Sr}$ include: chemical precipitation[4], solvent extraction[5], ion exchange[6], and adsorption[7]. So far, various adsorbents have been tested for sequestering of $\mathrm{Sr}^{2+}$ such as: zeolites[8], kaolinite[9], activated carbon[10], carbon nanotube[11], and magnetite[12]. Although some appreciable results have been achieved, there are still need for more efficient adsorbents in low cost and an environmentally friendly fabrication process.

DB18C6 is known to display strong and selective binding for alkaline earth metal divalent cations as well as alkali metal univalent cations. It was always used as the extractant for $\mathrm{Sr}^{2+}$ removing from the aqueous solutions[13]. Although it has good selectivity, extraction process is complex and difficult to operate.

In this paper, a novel adsorbent was prepared by DB18C6 grafted on the D564 resin. And the adsorption of $\mathrm{Sr}^{2+}$ from the aqueous solutions was investigated. The effects of the contact time, concentration of $\mathrm{Sr}^{2+}$ and $\mathrm{pH}$ of the solution were studied comprehensively.

\section{Experiments}

\subsection{Preparation of the adsorbent (D564-DB18C6)}

The reactions for the adsorbent synthesis were shown in Fig. 1.

(1) $10 \mathrm{~g} \mathrm{DB} 18 \mathrm{C} 6$ was immersed in $200 \mathrm{~mL} \mathrm{CHCl}_{3}$ and the mixture was stirred until DB18C6 was completely dissolved. $4 \mathrm{~mL} \mathrm{Br}_{2}$ was added to the mixture and the solution was heated up to $70^{\circ} \mathrm{C}$ and refluxing for $8 \mathrm{~h}$. Then the solvent was removed by a rotary evaporator after cooled. Finally, the white powder (Br-DB18C6) was dried at $30^{\circ} \mathrm{C}$.

(2) $12.5 \mathrm{~g}$ D564 resin was dispersed in $250 \mathrm{~mL} \mathrm{CHCl}_{3}$ and $20 \mathrm{~mL} 20 \% \mathrm{NaOH}$ was added by 
dropping. The mixture reacted at $70^{\circ} \mathrm{C}$ for $2 \mathrm{~h}$. After that, $5.0 \mathrm{~g} \mathrm{Br}$-DB18C6 was put into the mixture and refluxing for $24 \mathrm{~h}$ at $70^{\circ} \mathrm{C}$. When the reaction was complete, the resin was washed by $\mathrm{CHCl}_{3}$ and $\mathrm{EtOH}$ for several times and dried at $30^{\circ} \mathrm{C}$.

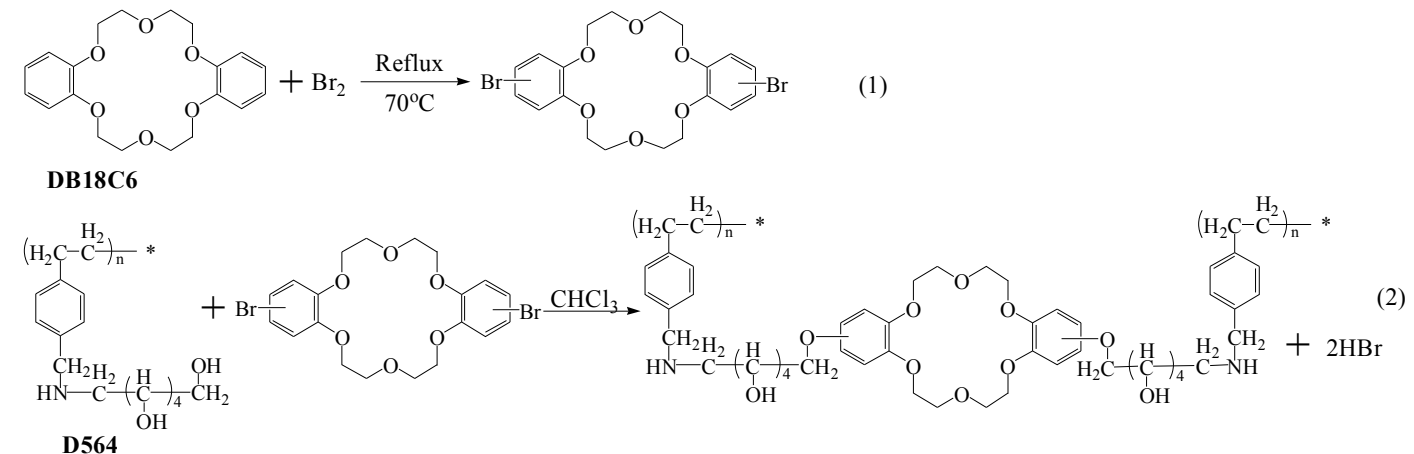

Fig.1 The reactions of the adsorbent synthesis

\subsection{Characterizations}

TG curves of samples were recorded on a TGDTA92 (SETARAM, France) apparatus in $\mathrm{N}_{2}$ with a heating rate of $10^{\circ} \mathrm{C} / \mathrm{min}$.

FTIR spectra of samples were taken following the usual $\mathrm{KBr}$ pellet technique on a Nexus FTIR spectrometer (Thermo-Nicolet, USA). Spectral resolution was $4 \mathrm{~cm}^{-1}$ and the spectra were obtained using 64 scans.

\subsection{Batch adsorption experiments}

In the batch adsorption experiments, $1.0 \mathrm{~g}$ adsorbent weighed into the $100 \mathrm{~mL}$ plastic bottles thoroughly mixed with $\mathrm{Sr}^{2+}$ solutions $(20 \mathrm{~mL})$ of the different initial concentrations, and $\mathrm{pH}$ was adjusted to the desired value. The suspension in flask was shaken for equilibrium in a thermostatic bath. After phase separation, the concentration of $\mathrm{Sr}^{2+}$ ion in the aqueous phase was analyzed by the ICS-1100 ionic chromatograph (Dionex Corporation). The amounts of metal ions adsorbed by the adsorbent were determined by mass balance. The adsorption capacity, $q(\mathrm{mmol} / \mathrm{g})$ can be calculated with the following equation:

$$
q=\frac{\left(C_{0}-C_{e}\right) V}{m}
$$

where $C_{0}$ and $C_{e}$ are the initial and equilibrium concentrations $(\mathrm{mmol} / \mathrm{L})$ of metal ions in solution, respectively. $V$ is the volume of solution $(\mathrm{mL})$ and $m$ is the weight of the adsorbents used $(\mathrm{g})$.

\section{Results and discussion}

\subsection{TG analysis}

The TG curves of DB18C6, Br-DB18C6, D564 resin and D564-DB18C6 are shown in Fig. 2 (a) and (b). As it can be seen from Fig. 2, they all start to decompose at $200^{\circ} \mathrm{C}$ and completed at $400^{\circ} \mathrm{C}$. However, the shape of the curves is not uniform with each other. The rate of weight loss of D564-DB18C6 is larger than D564 at 200 to $400^{\circ} \mathrm{C}$. It shows that DB18C6 is successfully loaded on D564.
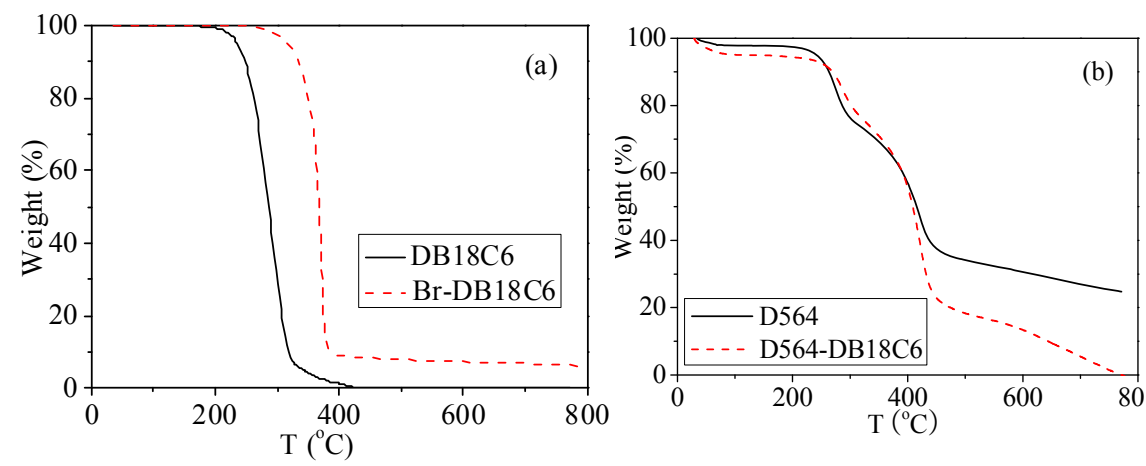

Fig. 2 TG curves 


\subsection{FTIR analysis}

The FTIR spectra ofDB18C6, Br-DB18C6, D564 resin and D564-DB18C6 are shown in Fig.3 (a) and (b), respectively. As it can be seen from Fig. 3 (a), a new band is appeared at $648 \mathrm{~cm}^{-1}$ which could be assigned to the structural C-Br groups in the Br-DB18C6. Moreover, the band at $741 \mathrm{~cm}^{-1}$ which is assigned to the 1,2-substituted benzene become weaker, and that indicated the reaction between $\mathrm{Br}_{2}$ and DB18C6 is achieved. From Fig.3 (b), several bands of D564 resin are disappeared after the reaction with Br-DB18C6.
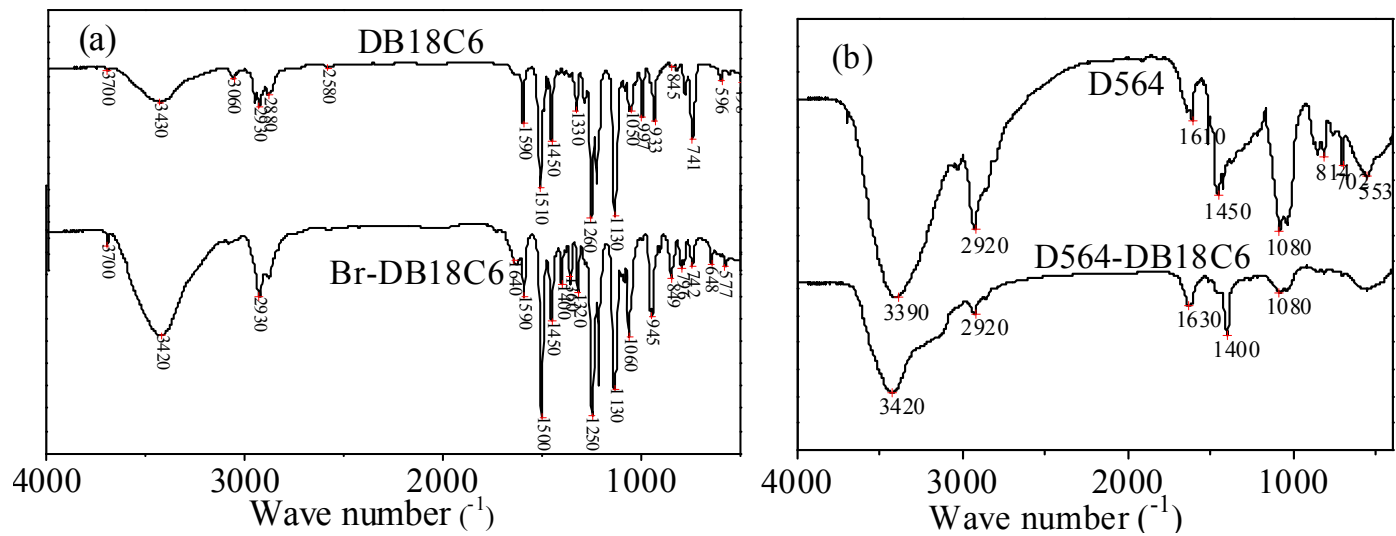

Fig.3 FTIRs of DB18C6, Br-DB18C6, D564 resin and D564-DB18C6

\subsection{Adsorption kinetics}

Fig. 4 illustrates the effect of contact time on adsorption. The most impressive of the kinetic data is that in the first $2 \mathrm{~h}$ at $25^{\circ} \mathrm{C}$ and $1 \mathrm{~h}$ at $50^{\circ} \mathrm{C}, \mathrm{Sr}^{2+}$ is removed more than $80 \%$, which is a rapid kinetic behavior. In the next time, the removal of $\mathrm{Sr}^{2+}$ continues but with lower rates, reaching the equilibrium after $6 \mathrm{~h}$ at $25^{\circ} \mathrm{C}$ and $2 \mathrm{~h}$ at $50^{\circ} \mathrm{C}$. The adsorption rate is faster than grapheme-oxide-magnetite adsorb $\mathrm{Sr} 2+$ and $\mathrm{Co} 2+$ ions, but it is slower than zeolite adsorb cesium and strontium[14].
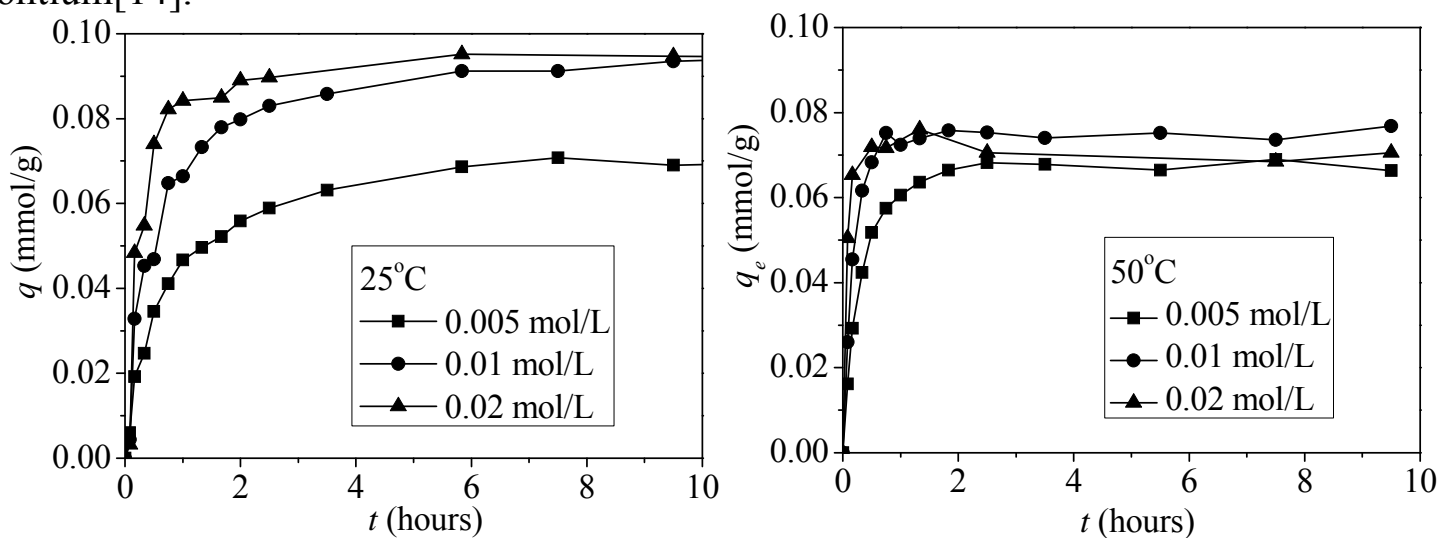

Fig. 4 Effect of immersion time on the adsorption of $\mathrm{Sr}^{2+}$

\subsection{Adsorption isotherms}

Fig. 5 depicts the adsorption isotherms of $\mathrm{Sr}^{2+}$ on D564-DB18C6 at 25 and $50^{\circ} \mathrm{C}$, respectively. In general, the equilibrium adsorption amount decreases with increasing temperature. The adsorption already reaches saturation when concentration of $\mathrm{Sr}^{2+}$ is $9 \mathrm{mmol} / \mathrm{L}$ at $50^{\circ} \mathrm{C}$. While the adsorption do not reach saturation when concentration of $\mathrm{Sr}^{2+}$ is $14.5 \mathrm{mmol} / \mathrm{L}$ at $25^{\circ} \mathrm{C}$. 

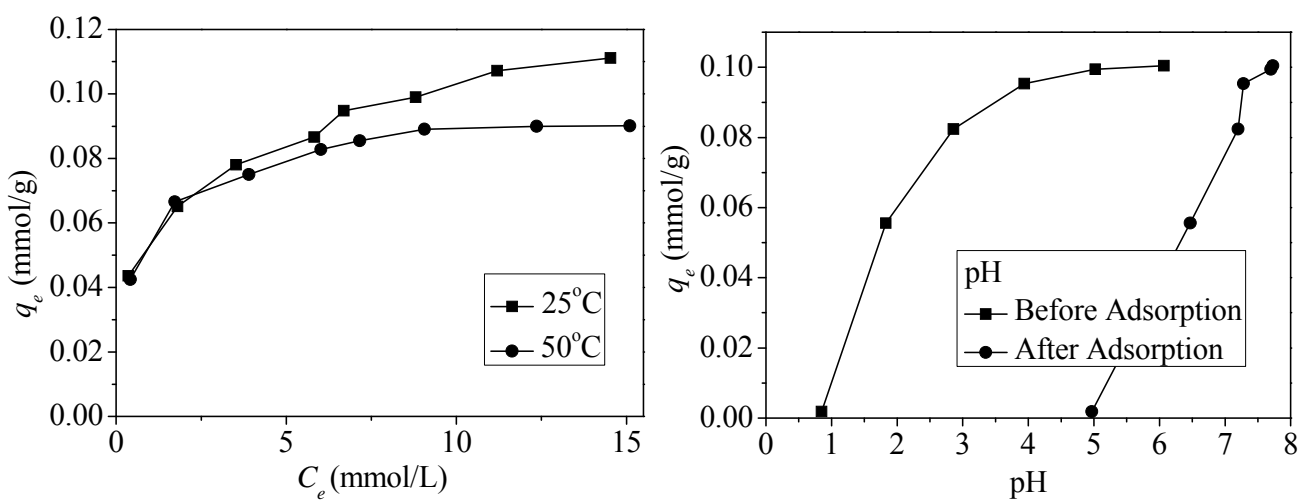

Fig.5 Adsorption isotherms

Fig. 6 Effect of the solution $\mathrm{pH}$ on the equilibrium adsorption amount.

\subsection{Effect of $\mathrm{pH}$}

The effect of the $\mathrm{pH}$ on the adsorption of $\mathrm{Sr}^{2+}$ is studied in the $\mathrm{pH}$ range from 0.85 to 6.07 before adsorption and the results are shown in Fig. 6. After adsorption, all the $\mathrm{pH}$ of the solutions become higher than before. And the highest adsorption amount is achieved at $\mathrm{pH} 5-6$ before adsorption and 7-8 after adsorption. This tendency is uniform with antimony silicate, alginate and grapheme oxide-magnetite adsorb strontium[15, 16].

\section{Conclusion}

A novel adsorbent was prepared by grafted the DB18C6 on D564 resins and used to adsorb $\mathrm{Sr}^{2+}$ from the solution. The TG curves and FTIR analysis exhibited that D564-DB18C6 by the reaction process. The results of the adsorption experiments showed that D564-DB18C6 has good adsorption properties for $\mathrm{Sr} 2^{+}$. The adsorption amount decreased with the increasing of temperature indicating that adsorption is exothermic nature. Solution $\mathrm{pH}$ has a significant effect on the equilibrium adsorption amount. Compared with acidic solution, in neutral solution, D564-DB18C6 could react with $\mathrm{Sr}^{2+}$ more easily. The highest adsorption amount is obtained at $\mathrm{pH} \sim 7$.

\section{Acknowledgments}

This work was financially supported by National Natural Science Foundation of China (51403229, U1407114, 21401209), the foundation of Knowledge Innovation Program of Chinese Academy of Sciences (kzcx2-ew-qn309), the Natural Science Foundation of Qinghai Province (2015-ZJ-933Q), "Western Light" Talents Training Program of Chinese Academy of Sciences, and the Talents Program of the Youth Innovation Promotion Association, Chinese Academy of Sciences (2013276).

\section{References}

[1] X. L. Gao, , Y. Q. Hu, T. Guo, et al. Comparative Study of the Competitive Adsorption of Mg, $\mathrm{Ca}$ and Sr Ions onto Resins. Adsorption Science \& Technology. Vol. 31 (2013) No. 1, p. 45-58.

[2] S. B. Yang, C. L. Chen, Y. Chen,, et al. Competitive Adsorption of Pb-II, Ni-II, and Sr-II Ions on Graphene Oxides: A Combined Experimental and Theoretical Study. Chempluschem. Vol. 80 (2015) No. 3, p. 480-484.

[3] M. Wang, L. Xu, J. Peng, et al. Adsorption and desorption of $\mathrm{Sr}$ (II) ions in the gels based on polysaccharide derivates. Journal of Hazardous Materials. Vol. 171 (2009) p. 820-826.

[4] R. W. Warrant, J. G. Reynolds, M. E. Johnson, et al. Removal of Sr-90 and Am-241 from concentrated Hanford chelate-bearing waste by precipitation with strontium nitrate and sodium permanganate. Journal of Radioanalytical and Nuclear Chemistry. Vol. 295 (2013) No. 2, p. 1575-1579. 
[5] J.Rais, S. Tachinori, E. Yoo, et al. Extraction of Radioactive Cs and Sr from Nitric Acid Solutions with 25,27-Bis(1-octyloxy)calix[4]-26,28-Crown-6 and Dicyclohexyl-18-Crown-6: Effect of Nature of the Organic Solvent. Separation Science and Technology. Vol. 50 (2015) No. 8, p. 1202-1212.

[6] Y. Ishikawa, S. Tsukimoto, K. S. Nakayama, et al. Ultrafine Sodium Titanate Nanowires with Extraordinary Sr Ion-Exchange Properties. Nano Letters. Vol. 15 (2015) No. 5, p. 2980-2984.

[7] A. Tayyebi, M. Outokesh, S. Moradi, et al. Synthesis and characterization of ultrasound assisted "graphene oxide-magnetite" hybrid, and investigation of its adsorption properties for $\mathrm{Sr}$ (II) and Co(II) ions. Applied Surface Science. Vol. 3532015 p. 350-362.

[8] A. Y. Lonin, A. P. Krasnopyorova. Studying Sorption Properties of a Mixture of Natural and Synthetic Zeolites in Respect of Radionuclides Cs-137 and Sr-90. Problems of Atomic Science and Technology. Vol.3 2009 p. 67-70.

[9] X. Chen, S. Peng, J. Wang, et al. Retention profile and kinetics characteristics of the radionuclide $90-\mathrm{Sr}$ (II) onto kaolinite. Journal of Radioanalytical and Nuclear Chemistry. Vol. 303 (2015) No. 1, p. 509-519.

[10] Y. Q. Lu, J. Yu, S. Cheng, et al. Magnetic composite of Fe3O4 and activated carbon as a adsorbent for separation of trace $\mathrm{Sr}$ (II) from radioactive wastewater. Journal of Radioanalytical and Nuclear Chemistry. Vol. 303 (2015) No. 3, p. 2371-2377.

[11] C. L. Chen, J. Hu, D. Shao, et al. Adsorption behavior of multiwall carbon nanotube/iron oxide magnetic composites for Ni(II) and Sr(II). Journal of Hazardous Materials. Vol. 164 (2009) No. 2-3, p. 923-928.

[12] A. Tayyebi, M. Outokesh, S. Moradi, et al. Synthesis and characterization of ultrasound assisted "graphene oxide-magnetite" hybrid, and investigation of its adsorption properties for $\operatorname{Sr}($ II) and Co(II) ions. Applied Surface Science. Vol. 353 (2015) p. 350-362.

[13] J. Heo. Theoretical Studies on Selectivity of Dibenzo-18-Crown-6-Ether for Alkaline Earth Divalent Cations. Bulletin of the Korean Chemical Society. Vol. 33 (2012) No. 8, p. 2669-2674.

[14] A. Tayyebi, M. Outokesh, S. Moradi, et al. Synthesis and characterization of ultrasound assisted "grapheneoxide-magnetite" hybrid, and investigation of its adsorptionproperties for $\operatorname{Sr}(\mathrm{II})$ and Co(II) ions. Applied Surface Science. Vol. 353 (2015) p. 350-362.

[15] H. Hong, J. Ryu, I. Park, et al. Investigation of the strontium ( $\operatorname{Sr}(\mathrm{II})$ ) adsorption of an alginate microsphere as a low-cost adsorbent for removal and recovery from seawater. Journal of Environmental Management. Vol. 165 (2016) pp. 263-270.

[16] L. Zhang, J. Wei, X. Zhao, et al. Adsorption characteristics of strontium on synthesized antimony silicate. Chemical Engineering Journal. Vol. 277 (2015) p. 378-387. 